\title{
Long-term survival of endometrioid endometrial cancer patients
}

\author{
Leszek Gottwald ${ }^{1,2}$, Piotr Pluta ${ }^{3}$, Janusz Piekarski ${ }^{3}$, Michał Spych ${ }^{4}$, Katarzyna Hendzel ${ }^{5}$, \\ Katarzyna Topczewska-Tylinska ${ }^{6}$, Dariusz Nejc ${ }^{3}$, Robert Bibik7 , Jerzy Korczyński, \\ Aleksandra Ciałkowska-Rysz ${ }^{1}$
}

\author{
1Palliative Care Unit, Chair of Oncology, Medical University of Lodz, Poland \\ ${ }^{2}$ Gynecological Cancer Outpatient Clinic, Regional Cancer Center, Copernicus Memorial \\ Hospital of Lodz, Poland \\ ${ }^{3}$ Department of Surgical Oncology, Chair of Oncology, Medical University of Lodz, Poland \\ 4Department of Radiotherapy, Chair of Oncology, Medical University of Lodz, Poland \\ ${ }^{5}$ Department of Histopathology, Madurowicz Memorial Hospital, Lodz, Poland \\ ${ }^{6}$ Department of Health Care Policy, Medical University of Lodz, Poland \\ 7 Department of Teleradiotherapy, Regional Cancer Center, Copernicus Memorial Hospital \\ of Lodz, Poland \\ ${ }^{8}$ Department of Fetal Medicine and Gynecology, $1^{\text {st }}$ Chair of Obstetrics and Gynecology, \\ Medical University of Lodz, Poland
}

Submitted: 28 November 2009

Accepted: 23 March 2010

Arch Med Sci 2010; 6, 6: 937-944

DOI: $10.5114 /$ aoms.2010.19305

Copyright (c) 2010 Termedia \& Banach

\begin{abstract}
Introduction: To establish risk factors for onset and progression of endometrioid endometrial cancer still remains the aim of scientists. The aim of the study was to determine disease-free survival (DFS) and overall survival (OS) in women with endometrioid endometrial cancer.

Material and methods: A retrospective review of 142 patients with endometrioid endometrial cancer after surgery treated with adjuvant radiotherapy and/or chemotherapy in the Regional Cancer Centre in Lodz between 2002 and 2004 was performed. Clinical and pathological data were correlated with clinical outcome and survival.

Results: In 3 patients (2.1\%) clinical progression was diagnosed during the treatment. In 23 patients (16.7\%) after primary remission, relapse was diagnosed 2-56 months after treatment. DFS and OS were $81.7 \%$ and $83.1 \%$ respectively. Better DFS significantly correlated with larger number of pregnancies $(>1)$, stage I of the disease and optimal surgery. Lower stage of disease, pelvic lymph node dissection, optimal surgery and depth of myometrial infiltration $\leq 50 \%$ were independent prognostic factors for better OS.

Conclusions: The results of our study provided significant evidence that early detection of endometrioid endometrial cancer enables optimal surgery. It reduces the indications for adjuvant therapy in stage I of the disease, and makes the prognosis significantly better. Other clinical and pathological factors such as numerous pregnancies, pelvic lymphadenectomy, and depth of myometrial infiltration, although important, are of less significance. Further prospective, randomized studies are necessary to prove the role of these factors.
\end{abstract}

Key words: endometrioid endometrial cancer, prognostic factors, disease-free survival, overall survival

\author{
Corresponding author: \\ Leszek Gottwald, MD, PhD \\ Palliative Care Unit \\ Chair of Oncology, \\ Medical University of Lodz \\ Ciołkowskiego 2 \\ 93-509 Lodz, Poland \\ Phone: +48 426895481 \\ Fax: +48 426895482 \\ E-mail: lgottwald@wp.pl
}




\section{Introduction}

Endometrial cancer is the most common gynaecological cancer, with an increasing incidence rate [1-3]. In the Polish female population endometrial cancer is ranked fourth in statistics of incidence of cancer, and eighth in terms of age-adjusted mortality [2]. In the United States the lifetime risk of endometrial cancer development is evaluated at $2.7 \%$ [3]. The increased incidence of endometrial cancer is likely to result from several factors including

Table I. Clinical and histological characteristics of the study group

\begin{tabular}{|c|c|c|}
\hline Variables & & $n=142, n(\%)$ \\
\hline \multirow[t]{2}{*}{ Age [years] } & $\leq 60$ & $77(54.2)$ \\
\hline & $>60$ & $65(45.8)$ \\
\hline \multirow[t]{3}{*}{ History of cancer } & no & $109(76.8)$ \\
\hline & patient & $5(3.5)$ \\
\hline & family & $28(19.7)$ \\
\hline \multirow[t]{2}{*}{ Pregnancies } & yes & $127(19.4)$ \\
\hline & no & $15(10.6)$ \\
\hline \multirow[t]{2}{*}{ Menopausal status } & premenopausal & $18(12.7)$ \\
\hline & postmenopausal & $124(87.3)$ \\
\hline \multirow{2}{*}{$\begin{array}{l}\text { Age at menopause } \\
\text { [years] }\end{array}$} & $\leq 50$ & $61(43.0)$ \\
\hline & $>50$ & $81(57.0)$ \\
\hline \multirow[t]{2}{*}{ Medical illness } & yes & $89(62.7)$ \\
\hline & no & $53(37.3)$ \\
\hline \multirow[t]{4}{*}{ Stage } & I & $96(67.6)$ \\
\hline & II & $18(12.7)$ \\
\hline & III & $26(18.3)$ \\
\hline & IV & $2(1.4)$ \\
\hline \multirow{2}{*}{$\begin{array}{l}\text { Depth of myometrial } \\
\text { infiltration }\end{array}$} & $\leq 50 \%$ & $72(50.7)$ \\
\hline & $>50 \%$ & $70(49.3)$ \\
\hline \multirow[t]{3}{*}{ Grade } & 1 & $71(50.0)$ \\
\hline & 2 & $54(38.0)$ \\
\hline & 3 & $17(12.0)$ \\
\hline \multirow[t]{5}{*}{ Surgery } & no or explorative & $2(1.4)$ \\
\hline & cytoreductive & $6(4.5)$ \\
\hline & optimal & $134(94.1)$ \\
\hline & $\mathrm{TAH}+\mathrm{BSO}$ & $40(28.1)$ \\
\hline & $\mathrm{TAH}+\mathrm{BSO}+\mathrm{PL}$ & $100(70.5)$ \\
\hline \multirow[t]{4}{*}{ Adjuvant treatment } & no & $6(4.2)$ \\
\hline & BT alone & $10(7.1)$ \\
\hline & $\mathrm{BT}+\mathrm{EBRT}$ & $122(85.9)$ \\
\hline & $C T \pm R T$ & $4(2.8)$ \\
\hline
\end{tabular}

TAH - total abdominal hysterectomy, BSO - bilateral salpingooophorectomy, PL - pelvic lymphadenectomy, CT - chemotherapy, $B T$ - vaginal brachytherapy, EBRT - external-beam pelvic radiotherapy, $R T$ - radiotherapy prolonged average human lifespan, high-caloric nutrition, improved health care system and living conditions [1]. Modern diagnostic methods, advances in surgical technique and high-efficacy adjuvant therapy, and increased health consciousness among women have resulted in relatively early detection of endometrial cancer (70-80\% stage I), which gives patients better prognosis [4-7].

Two types of endometrial cancer are distinguished in terms of clinical and pathological features. Type I (endometrioid carcinoma) is associated with hyperoestrogenism and endometrial hyperplasia (80-90\%). Type II (nonendometrioid carcinoma) is an oestrogenindependent malignancy, usually developing from atrophic endometrial tissues (10-20\%) [2, 3, 8]. Endometrioid carcinoma usually occurs in younger women, often obese or diabetic. Non-endometrioid carcinomas such as serous papillary, clear cell, squamous cell or undifferentiated type in most cases affect elderly women who are neither obese nor diabetic $[2,9]$. Several studies suggested more aggressive course and worse prognosis even in early stages of non-endometrioid endometrial cancer [2, 8-11]. The 5-year overall survival rate (OS) of endometrioid endometrial cancer patients ranges from $75 \%$ to $86 \%$. In contrast, only $35 \%$ of patients with non-endometrioid endometrial cancer survive 5 years from diagnosis $[3,9,10,12]$.

Several risk factors of endometrioid endometrial cancer have been identified, but the aetiology of the disease still remains unclear. However, progression from the pre-malignant form of intraendometrial neoplasia to endometrioid carcinoma seems to be well documented [1, 2, 12, 13]. To establish the risk factors of onset and progression of this disease still remains the aim of scientists. It may lead to more effective treatment in patients from the subgroup at high risk of failure and reduce the risk of overtreatment in the low-risk group.

The aim of the study was to determine prognostic factors for OS and disease-free survival (DFS) in women with endometrial cancer of endometrioid type.

\section{Material and methods}

Our study enrolled 142 consecutive endometrioid endometrial cancer patients with median age 60.2 years (range $41-82$ years), treated in the Regional Cancer Centre in Lodz between 2002 and 2004. Patients with incomplete follow-ups were excluded. All cancers were classified according to the new FIGO (International Federation of Gynecology and Obstetrics) classification 2009 [14]. Detailed clinical and pathological characteristics of the study group are presented in Table I.

Hundred forty one patients underwent surgical procedures outside the Regional Cancer Centre in 
Lodz. During surgery in 140 patients uterus and both adnexa were removed (TAH-BSO). In 99 $(69.7 \%)$ cases pelvic lymphadenectomy $(\mathrm{PL})$, and in 67 (47.2\%) cases appendectomy were additionally performed. Para-aortic lymph node sampling was not performed in these cases. Intraoperative procedures when TAH-BSO with complete resection of all visible cancer were regarded as optimal surgery. In one woman at stage IV no history of surgery was noted and in one patient the only explorative laparotomy was made. Afterwards adjuvant treatment was conducted in the Department of Gynaecologic Radiotherapy. Most women received adjuvant vaginal brachytherapy (BT) and external beam pelvic radiotherapy (EBRT). Additionally, selected patients were treated by chemotherapy (CT) and hormonal therapy (HT) (Table I). Afterwards the patients attended the Gynaecological Cancer Outpatient Clinic for followup. The clinical outcome of all patients during 5 years of observation was analysed. Personal, obstetric, oncological, clinical and pathological data were correlated with DFS and OS.

The DFS was defined as the period from primary surgery until relapse. OS was defined as the period from primary surgery until death or until completion of 5-year follow-up. Complete remission (CR) was defined as the disappearance of all signs of cancer in response to treatment. Progression of neoplastic disease was defined as the course of the disease as it becomes worse or spreads in the body. Relapse of cancer was defined as the return of signs and symptoms of cancer after a period of improvement.

To assess survival parameters Kaplan-Meier analysis was used [15] and survival curves were compared using the log-rank test [16]. Statistical analysis of survival was performed with the logrank test and Cox models. A $p$ value less than 0.05 was considered significant.

\section{Results}

Complete remission was observed in 116 out of $142(81.7 \%)$ patients during 5 years of follow-up. Clinical progression or relapse was diagnosed in $26(18.3 \%)$ patients. In 3 patients (2.1\%) with stage III or IV of the disease, clinical progression during primary treatment was diagnosed. Two of them had locoregional recurrence in the pelvis minor and dissemination of the disease after a few months was found. In 1 case bone and liver metastases were observed. These 3 patients were treated symptomatically and died during 5 months.

Relapse was observed in $23(16.2 \%)$ patients from 2 to 56 months (median 23.6, SD = 16.3) after CR achievement. Twenty-one out of 23 (91.3\%) patients with relapse died after 1 to 21 months (median 7.6) from diagnosis despite the consecutive line of treatment, and only 2 (8.7\%) patients with recurrent disease survived 5 years $(p<0.001)$. Detailed results of treatment are shown in Table II.

The DFS rate at 5 years of the entire group was $81.7 \%$. In univariate and multivariate analyses, larger number of pregnancies $(p=0.021, p=0.041$ ), clinical stage I $(p=0.002, p=0.04)$ and optimal surgery ( $p=0.01, p=0.028)$ significantly influenced DFS. In univariate and multivariate analyses, age at diagnosis, menopausal age and menopausal status, familial and patient cancer history, other diseases, pelvic lymph node dissection, depth of myometrial infiltration, grade of the tumour and type of adjuvant treatment in clinical stage I (no adjuvant and/or only vaginal brachytherapy [BT] vs. BT and external-beam pelvic radiotherapy [EBRT]) did not affect DFS. These results are summarized in Table III.

The OS rate at five years of the study group was 83.1\%. In univariate analysis, clinical stage I ( $p<0.001$; Fig. 2), optimal surgery $(p<0.001$; Fig. 1$)$, pelvic lymph node dissection $(p<0.001)$, depth of myometrial infiltration $\leq 50 \%(p<0.001$; Fig. 4$)$, and well or moderately differentiated tumour $(p=0.014$; Fig. 3) significantly influenced OS. Clinical stage I $(p=0.002)$, optimal surgery $(p=0.006)$, pelvic lymph node dissection $(p=0.015)$, and depth of myometrial infiltration $\leq 50 \%(p=0.02)$ were independent prognostic factors for OS in

Table II. Relapse of the disease

\begin{tabular}{|c|c|c|}
\hline \multicolumn{3}{|l|}{ Location of relapse } \\
\hline only locoregional & 3 & 13 \\
\hline only distant metastatic & 15 & 65.2 \\
\hline both locoregional and distant & 5 & 21.8 \\
\hline total & 23 & 100.0 \\
\hline \multicolumn{3}{|l|}{ Location of distant relapses at diagnosis } \\
\hline liver & 7 & 30.4 \\
\hline lungs & 7 & 30.4 \\
\hline bones & 3 & 13.0 \\
\hline $\begin{array}{l}\text { para-aortic and/or extraperitoneal } \\
\text { lymph nodes }\end{array}$ & 6 & 26.1 \\
\hline abdominopelvic layer & 3 & 13.0 \\
\hline central nervous system & 2 & 8.7 \\
\hline supraclavicular lymph nodes & 1 & 4.3 \\
\hline \multicolumn{3}{|l|}{ Treatment of relapses } \\
\hline only $\mathrm{CT}$ & 6 & 26.1 \\
\hline $\mathrm{S}+\mathrm{CT}$ & 2 & 8.7 \\
\hline only EBRT & 2 & 8.7 \\
\hline $\mathrm{BT}+\mathrm{EBRT}$ & 1 & 4.3 \\
\hline HT and symptomatically & 1 & 4.3 \\
\hline only symptomatically & 11 & 47.9 \\
\hline
\end{tabular}

S - surgery, CT - chemotherapy, BT - vaginal brachytherapy, EBRT - external-beam pelvic radiotherapy, HT - hormone therapy 
multivariate analysis. In univariate and multivariate analyses, age at diagnosis, larger number of pregnancies, menopausal age and menopausal status, familial and patient cancer history, concomitant diseases, and type of adjuvant treatment in clinical stage I (no adjuvant or only BT vs. BT and EBRT) were not significantly related to OS (Table III).

Table III. Disease-free survival (DFS) and 5-year overall survival (OS) - univariate and multivariate analysis

\begin{tabular}{|c|c|c|c|c|c|c|c|c|c|}
\hline \multirow[t]{2}{*}{ Patients } & & \multicolumn{4}{|c|}{ DFS } & \multicolumn{4}{|c|}{ OS } \\
\hline & & $n$ & $\%$ & $p^{1}$ & $p^{2}$ & $n$ & $\%$ & $p^{1}$ & $p^{2}$ \\
\hline TOTAL & & $116 / 142$ & 81.7 & - & - & 118 / 142 & 83.1 & - & \\
\hline \multirow[t]{2}{*}{ Age* [years] } & $\leq 60$ & $64 / 77$ & 85.7 & 0.61 & 0.64 & $65 / 77$ & 84.4 & 0.35 & 0.28 \\
\hline & $>60$ & $52 / 65$ & 80.0 & & & $53 / 65$ & 81.5 & & \\
\hline \multirow{3}{*}{$\begin{array}{l}\text { History } \\
\text { of cancer }\end{array}$} & no & $91 / 109$ & 83.5 & 0.77 & 0.11 & $92 / 109$ & 83.8 & 0.38 & 0.13 \\
\hline & patient & $3 / 5$ & 60.0 & & & $3 / 5$ & 60.0 & & \\
\hline & family & $23 / 28$ & 82.1 & & & $24 / 28$ & 85.7 & & \\
\hline \multirow[t]{2}{*}{ Pregnancies* } & yes & $104 / 127$ & 81.9 & 0.021 & 0.041 & $106 / 127$ & 83.5 & 0.13 & 0.09 \\
\hline & no & $12 / 15$ & 80.0 & & & $12 / 15$ & 80.0 & & \\
\hline \multirow{2}{*}{$\begin{array}{l}\text { Menopausal } \\
\text { status }\end{array}$} & premenopausal & $17 / 18$ & 94.4 & 0.41 & 0.90 & $17 / 18$ & 94.4 & 0.16 & 0.73 \\
\hline & postmenopausal & | $99 / 124$ & 79.8 & & & $101 / 124$ & 81.5 & & \\
\hline \multirow{2}{*}{$\begin{array}{l}\text { Age at } \\
\text { menopause* }\end{array}$} & $\leq 50$ & $47 / 61$ & 77.0 & 0.43 & 0.09 & 49 / 61 & 80.3 & 0.25 & 0.58 \\
\hline & $>50$ & $69 / 81$ & 85.2 & & & $69 / 81$ & 85.2 & & \\
\hline \multirow{2}{*}{$\begin{array}{l}\text { Medical } \\
\text { illness }\end{array}$} & yes & $75 / 89$ & 84.3 & 0.31 & 0.95 & $76 / 89$ & 85.4 & 0.46 & 0.67 \\
\hline & no & $41 / 53$ & 77.4 & & & $42 / 53$ & 79.2 & & \\
\hline \multirow{4}{*}{$\begin{array}{l}\text { Stage } \\
\text { [years] }\end{array}$} & I & $90 / 96$ & 93.8 & 0.002 & 0.040 & $91 / 96$ & 94.8 & $<0.001$ & 0.002 \\
\hline & II & $13 / 18$ & 72.2 & & & $13 / 18$ & 72.2 & & \\
\hline & III & $13 / 26$ & 50.0 & & & $14 / 26$ & 53.8 & & \\
\hline & IV & $0 / 2$ & 0 & & & $0 / 2$ & 0 & & \\
\hline \multirow{2}{*}{$\begin{array}{l}\text { Myometrial } \\
\text { infiltration }\end{array}$} & $\leq 50 \%$ & $69 / 72$ & 95.8 & 0.09 & 0.38 & $69 / 72$ & 95.8 & $<0.001$ & 0.02 \\
\hline & $>50 \%$ & $47 / 70$ & 67.1 & & & $49 / 70$ & 70.0 & & \\
\hline \multirow[t]{3}{*}{ Grade } & 1 & $62 / 71$ & 87.3 & 0.29 & 0.70 & $63 / 71$ & 88.7 & 0.014 & 0.09 \\
\hline & 2 & $43 / 53$ & 81.1 & & & $44 / 53$ & 83.0 & & \\
\hline & 3 & $11 / 18$ & 61.1 & & & $11 / 18$ & 61.1 & & \\
\hline \multirow[t]{5}{*}{ Surgery } & no or explorative & $0 / 2$ & 0 & 0.01 & 0.028 & $0 / 2$ & 0 & $<0.001$ & 0.006 \\
\hline & cytoreductive & $0 / 6$ & 0 & & & $0 / 6$ & 0 & & \\
\hline & optimal & $116 / 134$ & 86.6 & & & 118 / 134 & 88.1 & & \\
\hline & $\mathrm{TAH}+\mathrm{BSO}$ & $28 / 40$ & 70.0 & 0.13 & 0.24 & $29 / 40$ & 72.5 & $<0.001$ & 0.015 \\
\hline & $\mathrm{TAH}+\mathrm{BSO}+\mathrm{PL}$ & $88 / 100$ & 88.0 & & & $89 / 100$ & 89.0 & & \\
\hline \multirow{7}{*}{$\begin{array}{l}\text { Adjuvant } \\
\text { treatment }\end{array}$} & no & $5 / 6$ & 83.3 & $<0.001$ & 0.049 & $5 / 6$ & 83.3 & 0.67 & 0.35 \\
\hline & BT alone & $8 / 10$ & 80.0 & & & $8 / 10$ & 80.0 & & \\
\hline & $\mathrm{BT}+\mathrm{EBRT}$ & $103 / 122$ & 84.4 & & & $105 / 122$ & 86.1 & & \\
\hline & $\mathrm{CT}+/-\mathrm{RT}$ & $0 / 4$ & 0 & & & $0 / 4$ & 0 & & \\
\hline & & FIGO I** & & 0.23 & 0.37 & \multicolumn{2}{|c|}{ FIGO I** } & 0.11 & 0.07 \\
\hline & & FIGO II-III *** & & - & - & \multicolumn{2}{|c|}{ FIGO II-III *** } & - & - \\
\hline & & FIGO IV & & - & - & \multicolumn{2}{|c|}{ FIGO IV } & - & - \\
\hline
\end{tabular}

$p^{1}$ - univariate analysis, $p^{2}$ - multivariate analysis, TAH - total abdominal hysterectomy, BSO - bilateral salpingo-oophorectomy, $P L$ - pelvic lymphadenectomy, $C T$ - chemotherapy, BT - vaginal brachytherapy, EBRT-external-beam pelvic radiotherapy, $R T$ - radiotherapy, * in statistical analysis the exact values of all parameters in all patients were taken into account, ${ }^{* *}$ no adjuvant treatment or only BT VS. BT + EBRT, ${ }^{* * *}$ the treatment in these patients was BT +EBRT 


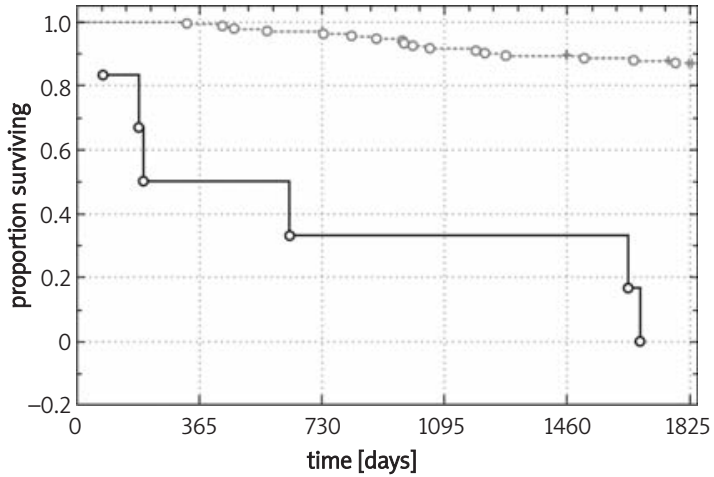

_ non-optimal surgery

...... optimal surgery

Figure 1. OS and radicality of surgery in endometrioid endometrial cancer.

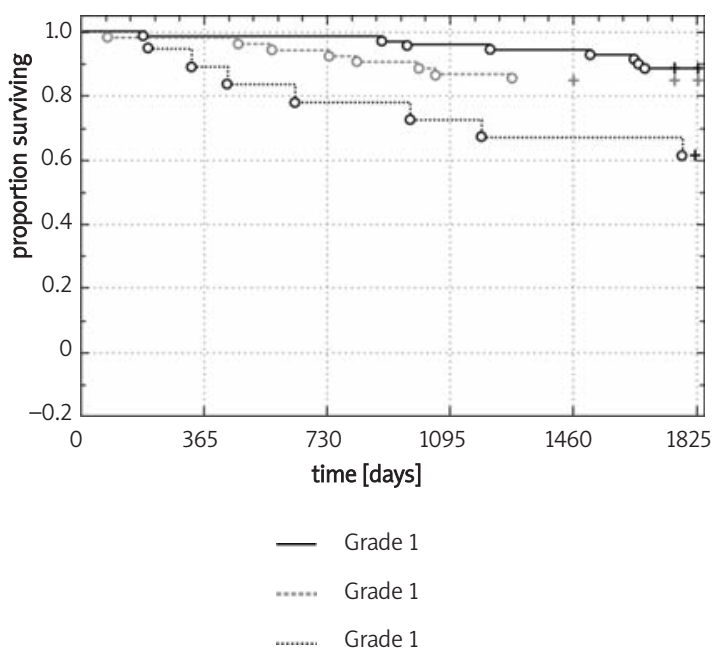

Figure 3. OS and grading of endometrioid endometrial cancer.

\section{Discussion}

In our study DFS and OS rates at five years were similar to data published by Hirai et al. [13], and were $81.7 \%$ and $83.1 \%$ respectively. In the last years there have been published numerous studies addressing the effects of different independent variables on the risk of development and prognosis of endometrioid endometrial cancer [1, 4-6, 11, 13, 17-23]. Age at diagnosis, age at menarche, parity, menopausal age, menstrual status, hormonal contraception, and hormone replacement therapy are well known risk factors for developing the cancer $[2,3,11,13,17,18]$, but as in our study, their

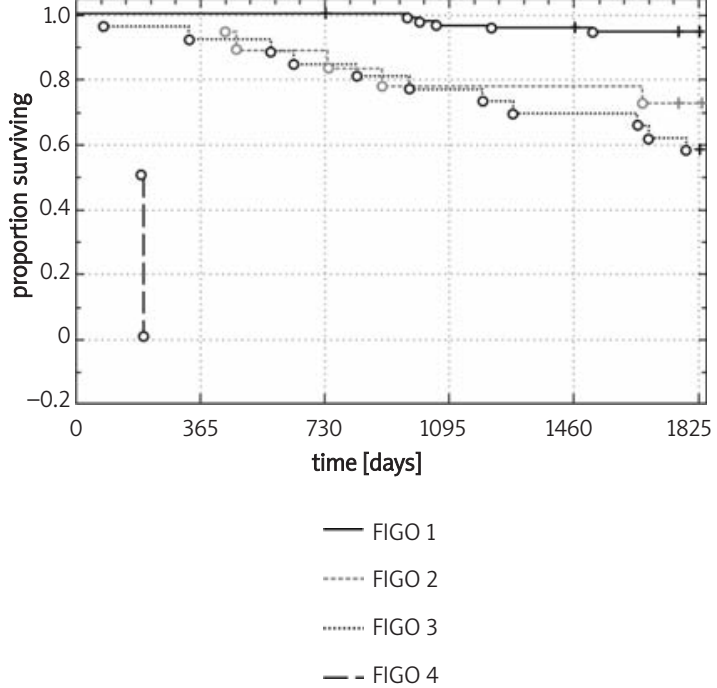

Figure 2. OS and staging of endometrioid endometrial cancer.

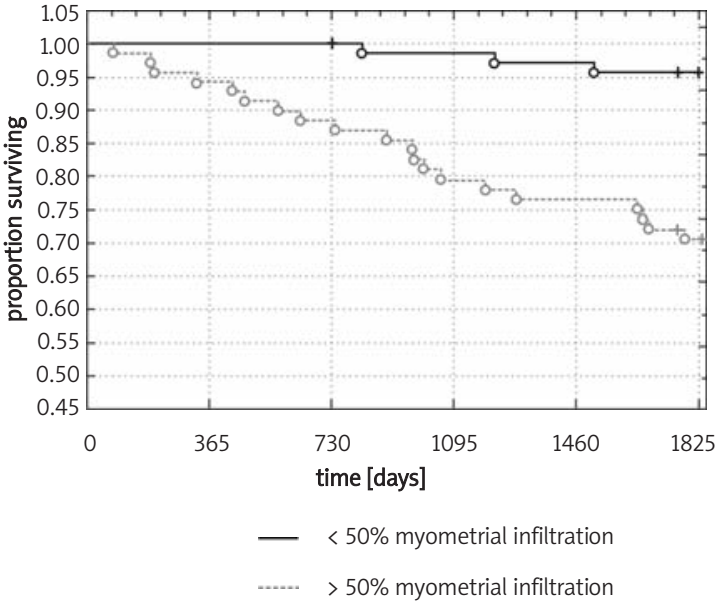

Figure 4. OS and myometrial infiltration in endometrioid endometrial cancer.

role as prognostic factors predicting survival remains controversial. Only higher number of pregnancies was correlated with better DFS, and our results match well with those of Alkbretsen et al. [19] reporting the relationship between obstetric history and prognosis in endometrioid endometrial cancer patients. They found better survival in parous than nulliparous women, and in the study the improved effect was strongest for women with the shortest time interval since the last childbirth. This interesting pattern may be a consequence of high progesterone levels, and interruption of the continued stimulation from oestrogen during pregnancy [3]. 
When planning the treatment of endometrioid endometrial cancer, complete pathological and clinical data predicting the course of the disease should be taken into consideration. It is generally known and confirmed in our results that the most important factor affecting survival is clinical stage $[12,13,24,25]$, which determines the mode of treatment, significantly predicting DFS and OS. As an example, Hirai et al. [13] in 286 patients reported the overall 5-year recurrence-free survival in stage I - 94\%, stage II - 71\% and stage III - 38\%.

The treatment of choice in operable endometrial cancer is surgery including minimally: TAH-BSO, sampling of peritoneal fluid and resection of all visible extra-uterine metastatic lesions, followed by adjuvant treatment [12, 26]. Previous studies showed that dissection of pelvic and para-aortal lymph nodes in all patients with endometrioid endometrial cancer remains controversial, because this procedure did not influence survival in clinical stage I [4, 12]. Ayhan et al. suggest instead performing pelvic lymphadenectomy in all patients because the most important prognostic factor in endometrial cancer is nodal involvement. Results of pelvic lymphadenectomy best determine the exact stage of patients, and allow tailoring of adjuvant therapy, and pelvic lymphadenectomy itself provides survival benefit and does not increase morbidity significantly [21]. In our study pelvic lymph node dissection correlated with longer OS in the whole group of patients as well. We conclude that, when technically feasible, pelvic lymph node dissection should be recommended in stage I-III disease. According to para-aortic lymphadenectomy, many authors have described para-aortic node biopsy as a non-therapeutic procedure [27], but Mariani et al. postulated its possible therapeutic role based on a selected group of 137 high-risk patients with endometrial cancer [28]. As para-aortic lymph node dissection is not routinely performed in all centres of gynaecological oncology, and this procedure is noted as technically demanding surgery, in our material it was not performed even in high-risk patients.

Although many studies have reported a significant negative correlation between grade of tumour and outcome $[8,9,29]$, our findings match well with those of Zaino et al. [30] and demonstrated tumour grade to be a less significant predictor of survival, with myometrial infiltration more important [24]. The results do not match well with those of Ayhan et al. [4], who in a group of 48 patients with endometrioid endometrial cancer in clinical stage II found high grade (G3) of the tumour to be a significant predictor of poor survival, and depth of myometrial infiltration was not related significantly to either DFS or OS. Interestingly, in the Gynecologic Oncology Group (GOG) study on
895 cases of endometrioid and adenosquamous carcinoma by Marrow et al., both depth of myometrial infiltration more than one-third and tumour grade 3 were demonstrated to be strong predictors of prognosis, extra-uterine disease and distant relapses in patients with stage I or II endometrial cancer [24]. Cirisano et al. [10] assessed the risk of positive pelvic nodes from $1 \%$ for tumours confined to the endometrium up to $25 \%$ for those with deep (> 50\%) myometrial infiltration. In our opinion the need of adjuvant treatment should be based on risk of relapse determined not only by stage, grade of tumour, and lymph vascular space involvement (LVSI), but in patients staged I additionally depth of myometrial infiltration as well.

Despite endometrial carcinoma being the most common gynaecological cancer in Poland, controversy exists regarding the indications for adjuvant radiotherapy, especially in clinical stage I [31]. Using surgical staging data according to the GOG [24] and the new FIGO classification from 2009 [14], in low-risk (IA G 1-2) endometrioid endometrial cancer adjuvant treatment is not necessary, because of the low recurrence rate calculated as $1-5 \%[5,21,22,26]$. Our results match well with the above cited recommendations, showing that radiotherapy did not improve DFS or OS in stage I disease. We also confirmed a positive correlation of adjuvant radiotherapy administered to patients with clinical stage II-III disease with DFS and OS. This matches well with the GOG recommendation [24] that when intermediate-risk (stage IA G 3, stage IB/2 G 1-3) or high-risk (stage III) endometrioid endometrial cancer is diagnosed, with calculated risk for relapse $10 \%$ and $14-42 \%$ respectively $[5,20$, 26], adjuvant therapy is recommended [4, 5, 20, 25, 31, 32]. The National Comprehensive Cancer Network (NCCN) Practice Guidelines [33] recommend vaginal brachytherapy (BT) and/or external-beam pelvic radiotherapy (EBRT) in intermediate-risk endometrial cancer patients, and BT and/or chemotherapy (CT) in patients with FIGO IB G3 and unfavourable risk factors (age > 60 years, positive LVSI, tumour size, lower uterine involvement) and II G3. Additionally, EBRT and/or CT is widely used as an adjuvant for intermediaterisk endometrial cancer, giving about $10 \%$ survival improvement, high locoregional control rates and acceptable toxicity. Although in the GOG122 trial [34] a superior effect on DFS of chemotherapy compared to whole abdominal irradiation for highrisk patients in stage III or IV with abdominal disease and residual nodules $\leq 2 \mathrm{~cm}$ was confirmed, different treatment schemes (EBRT and/or CT) in these patients are used worldwide [12,13]. Recurrent or metastatic endometrial tumours often respond to salvage treatment with cytotoxic agents, and 
nowadays systemic CAP (cisplatin-adriablastinendoxane) therapy as a post-surgical adjuvant treatment for high-risk endometrial cancer patients instead of radiotherapy is often used [35]. In our centre during 2002-2004 systemic CAP therapy was used mainly in relapses, and only in selected cases as primary adjuvant treatment.

Although the majority of patients with endometrial cancer are diagnosed with no evidence of extra-uterine spread [6], even in low-risk patients local relapses may develop. In our study, cancer progression during treatment or relapse occurred in $18.3 \%$ of patients. Local recurrences of endometrial cancer, similarly to cervical cancer, often occur early with evident symptoms but development of distant metastatic disease may cause difficulties in prompt diagnosis [23, 26, 36]. The previous study showed that in these patients survival rate is thought to be related to site of relapse as the most important factor, but also disease-free interval, and postoperative treatment as independent prognostic variables $[23,26]$. The response rates to systemic therapy reported in the literature in the range $10-78 \%$ are generally better than in our study, but this may be due to differences in the studied patient populations [6, 7]. The results of further treatment in our patients were similar to data published by Carey et al. [7], who found that median survival in patients with advanced endometrial cancer or distant recurrent disease rarely exceeds 1 year, despite systemic therapy.

Finally, the primary goal of a surveillance strategy in patients who have been treated for endometrial cancer is to facilitate the early detection of recurrent disease, but even in such cases the prognosis for patients should be assessed very carefully.

The results of our study provided significant evidence that early detection of endometrioid endometrial cancer enables optimal surgery. It reduces the indications for adjuvant therapy in stage I of the disease, and makes the prognosis significantly better. Other clinical and pathological factors such as numerous pregnancies, pelvic lymphadenectomy, and depth of myometrial infiltration, although important, are less significant. Further prospective, randomized studies are necessary to clarify the role of these factors.

\section{References}

1. Anastasiadis PG, Skaphida PG, Koutlaki NG, et al. Epidemiologic aspects of endometrial cancer in Thrace, Greece. Int J Gynecol Cancer 1999; 66: 263-72.

2. Purdie DM. Epidemiology of endometrial cancer. Reviews in Gynaecological Practice 2003; 3: 217-20.

3. Sonoda Y, Barakat RR. Screening and the prevention of gynecologic cancer: Endometrial cancer. Res Clin Obstet Gyn 2006; 20: 363-77.
4. Ayhan A, Taskiran C, Celik C, et al. Is there a survival benefit to adjuvant radiotherapy in high-risk surgical stage I endometrial cancer? Gynecol Oncol 2002; 86: 259-63.

5. Creutzberg CL, van Putten WL, Koper PC, et al. Survival after relapse in patients with endometrial cancer: results from a randomized trial. Gynecol Oncol 2003; 89: 201-9.

6. Preyer O, Obermair A, Formann E, et al. The impact of positive peritoneal washings and serosal and adnexal involvement on survival in patients with stage IIIA uterine cancer. Gynecol Oncol 2002; 86: 269-73.

7. Carey MS, Gawlik C, Kee-Fung MF, et al. Systematic review of systemic therapy for advanced or recurrent endometrial cancer. Gynecol Oncol 2006; 101: 158-67.

8. Sivridis E. Endometrial adenocarcinoma: an apostasy from early views. Gynecol Oncol 2004; 95: 772-7.

9. Alektiar KM, McKee A, Lin O, et al. Is there a difference in outcome between stage I-II endometrial cancer of papillary serous/clear cell and endometrioid FIGO grade 3 cancer? Int J Radiat Oncol Biol Phys 2002; 54: 79-85.

10. Cirisano FD, Robboy SJ, Dodge RK, et al. Epidemiopathologic and surgicopathologic findings of papillary serous and clear cell endometrial cancers when compared to endometrioid carcinoma. Gynecol Oncol 1999; 74: 385-94.

11. Pessini SA, Zettler CG, Wender MC, et al. Survival and prognostic factors of patients treated for Stage I to Stage III endometrial carcinoma in a reference cancer center in Southern Brazil. Eur J Gynaecol Oncol 2007; 28: 48-50.

12. Benedet $J$, Bender $\mathrm{H}$, Jones $\mathrm{H}$ 3rd, et al. FIGO staging classifications and clinical practice guidelines for gynaecological cancers. FIGO Committee on Gynecologic Oncology. Int J Gynaecol Obsetet 2000; 70: 209-62.

13. Hirai $M$, Hirono $M$, Oosaki $T$, et al. Prognostic factors relating to survival in uterine endometrioid carcinoma. Int J Gynecol Obstet 1999; 66: 155-62.

14. Pecorelli S, Denny L, Ngan H, et al. The new FIGO staging system for cancers of the vulva, cervix, endometrium and sarcomas. Gynecol Oncol 2009; 115: 325-8.

15. Kaplan EL, Meier P. Non-parametric estimation from incomplete observations. J Am Stat Assoc 1985; 53: 457-81.

16. Mantel N. Evaluation of survival data and two new rank order statistics arising in its consideration. Cancer Chemother Rep 1966; 50: 163-70.

17. Havrilesky LJ, Secord AA, O'Malley DM, et al. Multicenter analysis of recurrence and survival in stage IIIA endometrial cancer. Gynecol Oncol 2009; 114: 279-83.

18. Labastida R, Dexeus S, Fábregas R, et al. Endometrial cancer: factors affecting survival. Eur J Gynaecol Oncol 2003; 24: 381-3.

19. Albrektsen G, Heuch I, Wik E, Salvesen HB. Parity and time interval since childbirth influence survival in endometrial cancer patients. Int J Gynecol Cancer 2009; 19: 665-9.

20. Johnson N, Cornes P. Survival and recurrent disease after postoperative radiotherapy for early endometrial cancer: systematic review and meta-analysis. BJOG 2007; 114: 1313-20.

21. Ayhan A, Taskiran C, Celik C, Yuce K. The long-term survival of women with surgical stage II endometrioid type endometrial cancer. Gynecol Oncol 2004; 93: 9-13.

22. Fanning J. Long-term survival of intermediate risk endometrial cancer (stage IG3, IC, II) treated with full lymphadenectomy and brachytherapy without teletherapy. Gynecol Oncol 2001; 82: 371-4.

23. Sartori E, Laface B, Gadducci A, et al. Factors influencing survival in endometrial cancer relapsing patients: 
a Cooperation Task Force (CTF) study. Int J Gynecol Cancer 2003; 13: 458-65.

24. Morrow CP, Bundy BN, Kurman RJ, et al. Relationship between surgical-pathological risk factors and outcome in clinical stage I and II carcinoma of the endometrium: a Gynecologic Oncology Group Study. Gynecol Oncol 1991; 40: 55-65.

25. Watanabe Y, Kitanawa R, Aoki D, et al. Practice pattern for postoperative management of endometrial cancer in Japan: A survey of the Japanese Gynecologic Oncology Group. Gynecol Oncol 2009; 115: 456-9.

26. Fung-Kee-Fung M, Dodge J, Elit L, et al. Follow-up after primary therapy for endometrial cancer. A systematic review. Gynecol Oncol 2006; 101: 520-9.

27. Faught W, Krepart GV, Lotocki R, Heywood M. Should selective paraaortic lymphadenectomy be part of surgical staging for endometrial cancer? Gynecol Oncol 1994; 55: 51-5.

28. Mariani A, Webb MJ, Galli L, Podratz C. Potential therapeutic role of para-aortic lymphadenectomy in node-positive endometrial cancer. Gynecol Oncol 2000; 76: 348-65.

29. Abeler VM, Kjorstad KE. Endometrial adenocarcinoma in Norway: a study of a total population. Cancer 1991; 67: 3097-103.

30. Zaino RJ, Kurman RJ, Diana KL, Morrow CP. Pathologic models to predict outcome for women with endometrial adenocarcinoma. Cancer 1996; 77: 1115-21.

31. Macdonald OK, Sause WT, Lee RJ, et al. Adjuvant radiotherapy and survival outcomes in early-stage endometrial cancer: a multi-institutional analysis of 608 women. Gynecol Oncol 2006; 103: 661-6.

32. Bolukbasi Y, Demirci S, Ozsaran Z, et al. Postoperative radiotherapy in intermediate and high-risk Stage I endometrial cancer: analysis of prognostic factors and survival. Eur J Gynaecol Oncol 2008; 29: 505-10.

33. National Comprehensive Cancer Network. Clinical Practice Guidelines in Oncology V2 2009 (http://www.nccn.org/ professionals/physiciangls/PDF/uterine.pdf).

34. Randall ME, Filiaci VL, Muss H, et al. Randomized phase III trial of whole-abdominal irradiation versus doxorubicin and cisplatin chemotherapy in advanced endometrial carcinoma: a Gynecologic Oncology Group Study. J Clin Oncol 2006; 24: 36-44

35. Aoki Y, Watanabe M, Amikura T, et al. Adjuvant chemotherapy as treatment of high-risk stage I and II endometrial cancer. Gynecol Oncol 2004; 94: 333-9.

36. Gottwald L, Lech W, Sobotkowski J, et al. Transvaginal doppler sonography for assessment the response to radiotherapy in locally advanced squamous cervical cancer: a preliminary study. Arch Med Sci 2009; 5: 459-64. 